\title{
REVIEW \\ Effectiveness of transcranial direct current stimulation for the management of neuropathic pain after spinal cord injury: a meta-analysis
}

\author{
S Mehta ${ }^{1,2,3}$, A McIntyre ${ }^{1,2,3}$, S Guy $^{1,2,3}$, RW Teasell ${ }^{1,2,3}$ and E Loh ${ }^{1,2,3}$
}

Objectives: To conduct a systematic review and meta-analysis to examine the effect of transcranial direct current stimulation (tDCS) on reducing neuropathic pain intensity in individuals with spinal cord injury $(\mathrm{SCl})$.

Methods: Medline, CINAHL, EMBASE and PsycINFO databases were searched for all relevant articles published from 1980 to November 2014. Trials were included if (i) tDCS intervention group and a placebo control group were present; (ii) at least $50 \%$ of participants in the study had an SCl and there were at least three participants; (iii) participants were aged 18 years or older; and (iv) persistent pain for at least 3 months. Studies were excluded if: (i) the tDCS intervention group was compared with an active treatment group; (ii) there was insufficient reporting detail to enable pooling of data; and (iii) it was a nonclinical trial (that is, reviews, epidemiology, basic sciences). A standardized mean difference (SMD) \pm s.e. and $95 \%$ confidence interval (CI) was calculated for each outcome of interest and the results were pooled using a fixed or random effects model, as appropriate. Effect sizes were interpreted as: small $>0.2$, moderate $>0.5$, large $>0.8$.

Results: Five studies met inclusion criteria of which four were randomized controlled trials and one was a prospective controlled trial. The pooled analysis found a significant effect of tDCS on reducing neuropathic pain after SCI post treatment $(S M D=0.510 \pm 0.202$; 95\% Cl, 0.114-0.906; $P<0.012$ ); however, this effect was not maintained at follow-up (SMD $=0.353 \pm 0.272 ; 95 \% \mathrm{Cl},-0.179$ to $0.886 ; P<0.194)$. A reduction of 1.33 units on a 10 -item scale was observed post treatment. No significant adverse events were reported.

Conclusion: Meta-analytic results indicate a moderate effect of tDCS in reducing neuropathic pain among individuals with SCI; however, the effect was not maintained at follow-up. A mean pooled decrease of 1.33 units on a 10-item scale was found post treatment. Several factors were implicated in the effectiveness of tDCS in reducing pain. Due to the limited number of studies and lack of follow-up, more evidence is required before treatment recommendations can be made.

Spinal Cord (2015) 53, 780-785; doi:10.1038/sc.2015.118; published online 21 July 2015

\section{INTRODUCTION}

Chronic neuropathic pain (NP) can occur following lesions in the somatosensory nervous system ${ }^{1}$ and incidence rates range from $\sim 50^{2,3}$ to $75-81 \%{ }^{4,5}$ Several treatments have been proposed and used to manage NP, particularly pharmacological methods (for example, antidepressants, anticonvulsants, opioids) ${ }^{6,7}$ Despite multiple pharmacological options for pain, the majority of individuals fail to find relief; the refractory nature of NP is what makes it such a difficult condition to treat and to endure. Studies have shown that refractory $\mathrm{NP}$ is common post $\mathrm{SCI}^{8}$ and that few (4-6\%) obtain relief over the long-term. ${ }^{9}$ This situation has created interest in alternative neuromodulary stimulation approaches such as cranial electrotherapy stimulation, transcutaneous electrical nerve stimulation, spinal cord stimulation, repetitive transcranial magnetic stimulation (rTMS) and transcranial direct current stimulation (tDCS).

Many of these stimulation methods have demonstrated some effectiveness in pain relief. Deep brain stimulation (via intracranially implanted electrodes) has been performed with effectiveness, ${ }^{10}$ however, given the invasiveness of the procedure, less intrusive treatments such as rTMS and tDCS, may be preferable due to a lower risk of complications. rTMS and TDCS can be applied without invasive methods and use either brief high-intensity or magnetic pulses or lowintensity electrical currents to stimulate brain matter, respectively. ${ }^{11}$ Compared with rTMS, tDCS offers advantages in longer-lasting modulatory effects in cortical functioning, ease of administration, inexpensive cost and reliable sham-controlled conditions. ${ }^{12}$

The mechanism by which tDCS is able to relieve pain is not entirely known; however, it is believed that tDCS may improve pain processing due to a modulatory effect on central pathways, the same pathways targeted by antidepressant pharmacotherapy. ${ }^{11,13-14}$ Given the refractory nature of NP in a post SCI population, it is important to uncover new modalities for treatment. Until recently, there were too few studies evaluating the effectiveness of tDCS for NP among individuals post SCI; however, an increase in interest in this field

${ }^{1}$ Aging, Rehabilitation, and Geriatric Care, Lawson Health Research Institute, London, Ontario, Canada; ${ }^{2}$ Western University, London, Ontario, Canada and ${ }^{3}$ Parkwood Institute, London, Ontario, Canada

Correspondence: Professor S Mehta, Parkwood Institute, Lawson Health Research Institute, Main Building Room B3025, 550 Wellington Road, London N6C 0A7, Ontario, Canada.

E-mail: swati.mehta@sjhc.london.on.ca

Received 26 February 2015; revised 3 June 2015; accepted 14 June 2015; published online 21 July 2015 
has led to several new studies to assess. Therefore, it was our objective to perform a meta-analysis examining the effectiveness of tDCS in treatment of NP among those with SCI.

\section{MATERIALS AND METHODS}

\section{Literature search strategy}

Potentially relevant articles were identified by a literature search from 1980 to November 2014 using multiple databases (MEDLINE, CINAHL, EMBASE, PsycInfo). Grey literature including Cochrane Library was consulted to evaluate previous review articles. Previous reviews were cross-referenced for any primary studies missed through our search. Key words used included: 'spinal cord injury,' 'paraplegia,' 'quadriplegia,' 'tetraplegia,' pain, 'transcranial direct current stimulation' and 'tDCS.' References of retrieved articles were also hand searched to find additional articles that may have been missed in the database search.

\section{Study selection and quality}

Titles, abstracts and full texts were screened by two independent reviewers for eligibility. Any discrepancies regarding study eligibility were reconciled by a third independent reviewer. Studies were selected for analysis if the following criteria were met: (i) tDCS intervention group and a placebo control group were present; (ii) at least $50 \%$ of participants in the study had an SCI and there were at least three participants; (iii) participants were aged 18 years or older; and (iv) persistent pain for at least 3 months. Studies were excluded if: (i) the tDCS intervention group was compared with an active treatment group; (ii) there was insufficient reporting detail to enable pooling of data; and (iii) it was a nonclinical trial (that is, reviews, epidemiology and basic sciences).

Study quality was assessed by two independent reviewers using the Physiotherapy Evidence Database (PEDro) scoring system. ${ }^{15}$ Rare scoring discrepancies were resolved by a third independent reviewer. The PEDro tool consists of 11 questions with a maximum score of 10 . The following criteria were used for rating the methodological quality of a study: 9 to 10, excellent; 6 to 8 , good; 4 to 5 , fair; and $<4$ poor. ${ }^{16}$ All studies were included in the analysis regardless of study quality.

\section{Data analysis}

Data on each study's design, participant characteristics, intervention(s), outcome(s) and adverse event(s) were extracted from each of the selected studies. Pooled analyses were conducted for the primary outcome of pain intensity post-treatment and at follow-up. Treatment effect was reported as a standardized mean difference $(\mathrm{SMD}) \pm$ s.e. and $95 \%$ confidence interval $(\mathrm{CI})$ using the software Comprehensive Meta-Analysis (version 2, Biostat Inc., Englewood, NJ, USA, 2007). Heterogeneity between studies was measured by the $I^{2}$ statistic. An $I^{2}$ value exceeding $50 \%$ was used as the threshold to identify significant statistical heterogeneity. A fixed effects model was used when the threshold for heterogeneity was not reached, and a random effects model was used when it was exceeded. No substantial statistical heterogeneity was evident for post treatment time point $I^{2}=10.25 \%$ or at follow-up $I^{2}=27 \%$; hence, a fixed effects model was used to pool data from both time points. Cohen's criteria ${ }^{17}$ were used to interpret resulting effect sizes: small $>0.2$, moderate $>0.5$, large $>0.8$. To enhance clinical relevance, effect sizes were converted into their original units using a standardized technique. ${ }^{18}$

\section{RESULTS}

Study selection and quality

Five studies met inclusion criteria (Figure 1). These included two double-blinded randomized controlled trials (RCTs), ${ }^{12,19}$ two doubleblinded crossover RCTs, ${ }^{20,21}$ and one prospective controlled trial. ${ }^{22}$ The methodological quality was excellent for the four RCTs, ${ }^{12,19-21}$ whereas the prospective controlled trial ${ }^{22}$ was fair in quality (Table 1).

\section{Study design}

All five studies involved an active tDCS group and a sham tDCS group. Soler and colleagues ${ }^{19}$ randomized individuals into four groups: tDCS, tDCS plus visual illusion, visual illusion only and sham tDCS. To examine objective data, data from visual illusion groups were excluded so that only data from the active tDCS and sham group were examined. Both Fregni et al. ${ }^{12}$ and Wrigley et al. ${ }^{21}$ randomized participants to receive sham or active tDCS for five consecutive days. Participants in the tDCS group received a constant current of $2 \mathrm{~mA}$ for $20 \mathrm{~min}$, whereas participants in the sham tDCS group received $10 \mathrm{~s}$ of stimulation after which the stimulator was turned off. Wrigley et al. ${ }^{21}$ provided a 4-week washout period before participants were crossed over to the alternative protocol. Participants in the Soler et al. ${ }^{19}$ study received treatment (active or sham) for 10 days over a 2-week period. A constant current of $2 \mathrm{~mA}$ for $20 \mathrm{~min}$ was delivered to the tDCS group while the sham group received stimulation for $30 \mathrm{~s}$ after which it was turned off. Ngernyam et al. ${ }^{20}$ randomized individuals into active or sham $\mathrm{tDCS}$ groups with a 1-week washout period before individuals were crossed over to the alternative protocol. Individuals received a single session of $2 \mathrm{~mA}$ active or sham treatment for $20 \mathrm{~min} .{ }^{20}$ Yoon et al. $^{22}$ also provided participants with twice daily treatment of a total of 20 treatment sessions over a 2 -week period. Similar to the other studies, a $2 \mathrm{~mA}$ current was applied for $20 \mathrm{~min}$ per session to the active tDCS group, whereas the sham group only received the current for $10 \mathrm{~s}$. All studies placed the anodal stimulation onto the primary motor cortex (M1).

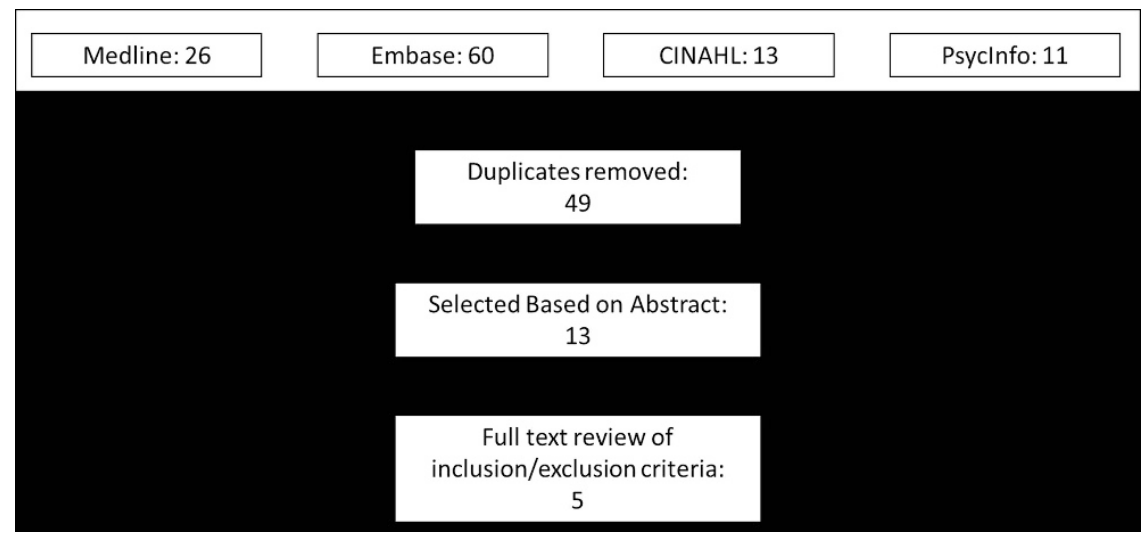

Figure 1 Study Selection Process. 
Patient characteristics

Table 1 presents detailed information on patient characteristics. Studies involved small sample sizes ranging from 10 to 20 participants. Participants were predominantly males in all studies and the average age ranged from 35 to 56 years. Duration of NP ranged from 3.7 to 15.8 years. Four studies included participants with complete and incomplete injuries. ${ }^{12,19,20,22}$ Wrigley et al. ${ }^{21}$ included only participants with complete injuries. Four studies included individuals with cervical

\section{Table 1 Study characteristics}

\begin{tabular}{|c|c|c|c|}
\hline $\begin{array}{l}\text { Study name, design } \\
\text { quality, } N\end{array}$ & Average age duration of pain & $\begin{array}{l}\text { Severity of injury; } \\
\text { level of injury }\end{array}$ & Treatment protocol \\
\hline $\begin{array}{l}\text { Fregni et al. }{ }^{12} \\
\text { double blinded RCT } \\
\text { PEDro }=9 N=17\end{array}$ & $\begin{array}{l}\text { Age }=35.4 \mathrm{yrs} \\
\text { Duration of Pain }=3.7 \mathrm{yrs}\end{array}$ & $\begin{array}{l}\text { Severity of Injury = } 11 \\
\text { complete, } 6 \text { incomplete } \\
\text { Level of injury = } 9 \text { cervical, } 8 \\
\text { below cervical }\end{array}$ & $\begin{array}{l}\text { Patients were given sham or active tDCS treatment for } 5 \text { consecutive days } \\
\text { and followed up for } 16 \text { days. A constant current of } 2 \mathrm{~mA} \text { was applied for } \\
20 \mathrm{~min} \text {. For sham the stimulator was turned off after } 10 \mathrm{~s} \text { of stimulation. }\end{array}$ \\
\hline $\begin{array}{l}\text { Soler et al. }{ }^{19} \\
\text { Double blinded RCT } \\
\text { PEDro }=9 \quad N=20\end{array}$ & $\begin{array}{l}\text { Age }=42.95 \text { yrs } \\
\text { Duration of pain }=8.6 \text { yrs } \\
\text { (treatment); } 5.6 \text { yrs(control) }\end{array}$ & $\begin{array}{l}\text { Severity of injury }=16 \\
\text { complete, } 4 \text { incomplete } \\
\text { Level of injury }=15 \text { thoracic, } \\
5 \text { cervical }\end{array}$ & $\begin{array}{l}\text { Participants were randomized to tDCS or sham groups. A constant current of } \\
2 \mathrm{~mA} \text { intensity was applied for } 20 \text { min. For sham stimulation the electrodes } \\
\text { were placed in the same position; however, the stimulator was turned off } \\
\text { after } 30 \mathrm{~s} \text { of simulation so subjects felt the initial itching sensation. Each } \\
\text { patient received } 10 \text { treatment sessions, } 20 \text { min each during a period of } \\
2 \text { weeks. }\end{array}$ \\
\hline $\begin{array}{l}\text { Wrigley et al. }{ }^{21} \\
\text { Double blinded cross- } \\
\text { over RCT } \\
\text { PEDro = } 9 \quad N=10\end{array}$ & $\begin{array}{l}\text { Age }=56.1 \mathrm{yrs} \\
\text { Duration of pain }=15.8 \mathrm{yrs}\end{array}$ & $\begin{array}{l}\text { Severity of injury }=10 \text { com- } \\
\text { plete } \\
\text { Level of injury }=10 \text { thoracic }\end{array}$ & $\begin{array}{l}\text { Participants were randomized to tDCS or sham. One } 20 \text { min treatment } \\
\text { session was delivered each day for } 5 \text { consecutive days. A } 4 \text { week washout } \\
\text { period took place before crossover to sham or treatment. }\end{array}$ \\
\hline $\begin{array}{l}\text { Yoon et al. } .^{22} \\
\text { PCT } \\
\text { PEDro }=5 \quad N=16\end{array}$ & $\begin{array}{l}\text { Age }=44.9 y r s \\
\text { Duration of pain }=2.3 y r s\end{array}$ & $\begin{array}{l}\text { Severity of injury = } 10 \text { com- } \\
\text { plete, } 6 \text { incomplete } \\
\text { Level of injury = } 8 \text { cervical, } 8 \\
\text { thoracic }\end{array}$ & $\begin{array}{l}\text { Subjects were randomized to tDCS or sham. } 2 \text { mA current was applied for } \\
20 \text { min. Each patient received } 20 \text { treatments over } 2 \text {-week period, } 2 x \text { daily } \\
\text { with an interval of at least } 4 \text { hours in between. During sham procedure } \\
\text { stimulator was turned off after } 10 \text { s. }\end{array}$ \\
\hline $\begin{array}{l}\text { Ngernyam et al. }{ }^{20} \\
\text { Double blind cross- } \\
\text { over RCT } \\
\text { PEDro }=9 N=20\end{array}$ & $\begin{array}{l}\text { Age }=44.50 \mathrm{yrs} \\
\text { Duration of pain }=4.2 \mathrm{yrs}\end{array}$ & $\begin{array}{l}\text { Severity of injury }=9 \text { com- } \\
\text { plete, } 11 \text { incomplete } \\
\text { Level of injury = } 12 \text { thoracic, } \\
7 \text { cervical, } 1 \text { lumbar }\end{array}$ & $\begin{array}{l}\text { Participants were randomized to tDCS or sham, } 1 \text { treatment at } 2 \mathrm{~mA} \text { for } \\
20 \mathrm{~min} \text {, and then } 4 \text { week washout and switched to other intervention }\end{array}$ \\
\hline
\end{tabular}

Abbreviations: PCT, prospective controlled trial; PEDro, Physiotherapy Evidence Database (PEDro) scoring system; RCT, randomized controlled trial; yrs, years.

a Studyname

$\begin{array}{lrrrrrrr} & \begin{array}{c}\text { Std diff } \\ \text { in means }\end{array} & \begin{array}{c}\text { Standard } \\ \text { error }\end{array} & \text { Variance } & \begin{array}{c}\text { Lower } \\ \text { limit }\end{array} & \begin{array}{c}\text { Upper } \\ \text { limit }\end{array} & \text { Z-Value } & \text { p-Value } \\ \text { Fregni et al. 2006 } & 1.551 & 0.573 & 0.328 & 0.428 & 2.674 & 2.707 & 0.007 \\ \text { Soler et al. 2010 } & 0.242 & 0.449 & 0.201 & -0.638 & 1.121 & 0.538 & 0.590 \\ \text { Wrigley et al. 2013 } & -1.214 & 0.487 & 0.237 & -2.167 & -0.260 & -2494 & 0.013 \\ \text { Yoon et al. 2014 } & 0.614 & 0.528 & 0.278 & -0.420 & 1.648 & 1.163 & 0.245 \\ \text { Ngernyamet al. 2014 } & 1.093 & 0.339 & 0.115 & 0.428 & 1.757 & 3.224 & 0.001 \\ & 0.510 & 0.202 & 0.041 & 0.114 & 0.906 & 2.526 & 0.012\end{array}$

b Studyname

\begin{tabular}{lrc} 
& $\begin{array}{c}\text { Std diff } \\
\text { in means }\end{array}$ & \multicolumn{1}{c}{$\begin{array}{c}\text { Standard } \\
\text { error }\end{array}$} \\
Fregni et al. 2006 & 0.822 & 0.527 \\
Soler et al. 2010 & 0.052 & 0.447 \\
Wrigleyet al. 2013 & 0.316 & 0.450 \\
& 0.353 & 0.272
\end{tabular}

Statistics for each study

$\begin{array}{rccrr}\text { Variance } & \begin{array}{c}\text { Lower } \\ \text { limit }\end{array} & \begin{array}{c}\text { Upper } \\ \text { limit }\end{array} & \begin{array}{r}\text { Z-Value } \\ \text { p-Value }\end{array} \\ 0.277 & -0.210 & 1.855 & 1.561 & 0.118 \\ 0.200 & -0.825 & 0.928 & 0.116 & 0.908 \\ 0.203 & -0.566 & 1.198 & 0.703 & 0.482 \\ 0.074 & -0.179 & 0.886 & 1.300 & 0.194\end{array}$

Std diff in means and $95 \% \mathrm{Cl}$

$\mid$

$-4.00$

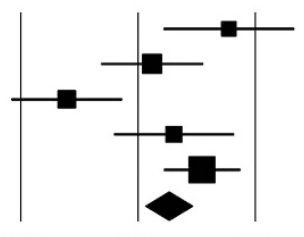

2.00

Active tDCs
Std diff in means and $95 \% \mathrm{Cl}$

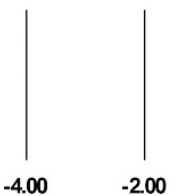

Sham tDCS

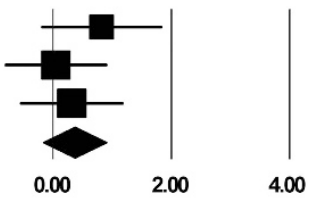

Active tDCS

Figure 2 Primary analysis. (a) Forest plot comparing the effects tDCS on reducing pain intensity post treatment. Std diff: standard difference. (b). Forest plot comparing the effects tDCS on reducing pain intensity at follow-up. Std diff: standard difference. 
and thoracic injuries, ${ }^{12,19,20,22}$ whereas only participants with thoracic injury were included in the study by Wrigley et al. ${ }^{21}$ All studies included only those participants with traumatic SCI.

Fregni et al. ${ }^{12}$ and Yoon et al. ${ }^{22}$ recruited participants with pain for at least 3 months, whereas three studies ${ }^{19-21}$ included participants with NP for at least 6 months. Assessment of pain was conducted through a clinical interview in all five studies. Four studies recruited only participants that were refractory to previous pharmacological treatment. ${ }^{12,19,20,22}$ Only two studies excluded participants based on high levels of depressive symptoms. ${ }^{19,20}$ All five studies allowed participants to remain on their current medication routine. Three studies assessed allocation concealment and blinding; the authors found no correlation between correct and incorrect responses in predicting group allocation. ${ }^{12,19,21}$

\section{Effectiveness}

The pooled analysis found a significant effect of tDCS on reducing neuropathic pain post treatment among individuals with SCI (SMD = $0.510 \pm 0.202 ; 95 \% \mathrm{CI}, 0.114-0.906 ; \quad P<0.012)$; however, this was not maintained at follow-up $(\mathrm{SMD}=0.353 \pm 0.272 ; 95 \% \mathrm{CI}$, $-0.179-0.886$; $P<0.194$; Figure 2). A mean pooled decrease of 1.33 units on a 10-item Visual Analog Scale (VAS) was observed post treatment, with a decrease of only 0.50 units at follow-up. No significant improvement in psychosocial outcomes including depression $^{12,21,22}$ and anxiety ${ }^{19}$ were observed. One study found a significant improvement in mood compared with placebo post treatment; however, this was not maintained at follow-up. ${ }^{19}$

Subanalysis found a significant effect of tDCS among those studies that excluded patients with depression $(\mathrm{SMD}=0.784 \pm 0.271 ; 95 \% \mathrm{CI}$, $0.253-1.314 ; P<0.004)$, whereas studies that included participants with depressive symptoms did not reach significance (Figure 3). Studies with participants with an average pain duration of $<5$ years resulted in significant improvement post tDCS treatment $(\mathrm{SMD}=$ $1.072 \pm 0.255 ; 95 \% \mathrm{CI},-0.428-1.757 ; P<0.001)$, whereas those with $>5$ years did not. A sub-analysis examining duration of treatment found that those studies that provided treatment for $<1$ week resulted in significant reduction in pain intensity among their participants $(\mathrm{SMD}=0.570 \pm 0.250 ; 95 \% \mathrm{CI}, 0.080-0.1 .061 ; P<0.001)$. However, those studies that provided treatment for $>1$ week did not result in significant improvement. Adverse events included mild headaches and itching under electrodes.

\section{DISCUSSION}

This meta-analysis is the first study to conduct a pooled analysis on the effectiveness of tDCS for the treatment of neuropathic pain after SCI. On the basis of five studies, the authors found a moderate effect a

\begin{tabular}{|c|c|c|c|c|c|c|c|c|}
\hline \multirow{2}{*}{$\begin{array}{l}\text { Group by } \\
\text { Outcome }\end{array}$} & \multirow[t]{2}{*}{ Study name } & \multicolumn{7}{|c|}{ Statistics for each study } \\
\hline & & $\begin{array}{l}\text { td diff } \\
\text { means }\end{array}$ & $\begin{array}{l}\text { Standard } \\
\text { error }\end{array}$ & Variance & $\begin{array}{c}\text { Lower } \\
\text { limit }\end{array}$ & $\begin{array}{l}\text { Upper } \\
\text { limit }\end{array}$ & Z-Value & p-Valu \\
\hline Exclude & Soler et al. 2010 & 0.242 & 0.449 & 0.201 & -0.638 & 1.121 & 0.538 & 0.5 \\
\hline Exclude & Ngemyam et al. 2014 & 41.093 & 0.339 & 0.115 & 0.428 & 1.757 & 3.224 & 0.00 \\
\hline Exclude & & 0.784 & 0.271 & 0.073 & 0.253 & 1.314 & 2.897 & 0.00 \\
\hline Include & Fregni et al. 2006 & 1.551 & 0.573 & 0.328 & 0.428 & 2.674 & 2.707 & 0.00 \\
\hline Include & Wrigley et al. 2013 & -1.214 & 0.487 & 0.237 & -2.167 & -0.260 & -2.494 & 0.0 \\
\hline Include & Yoon et al. 2014 & 0.614 & 0.528 & 0.278 & -0.420 & 1.648 & 1.163 & 0.2 \\
\hline Include & & 0.166 & 0.303 & 0.092 & -0.429 & 0761 & 0.547 & \\
\hline
\end{tabular}
Group by Time point

$<=5$ years

$<=5$ years

$<=5$ years

$<=5$ years

$>5$ years

$>5$ years

$>5$ years
Study name

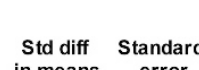

Statistics for each study

Fregni et al. $2006 \quad 1.551$

Yoon et al. $2014 \quad 0.614$

Ngemyam et al. 20141.093

1.072

Soler et al. $2010 \quad 0.242$

Wrigley et al. $2013 \quad-1.214$

$-0.427$

$$
0.573
$$

0.528

0.339

0.255

0.449

0.487

0.330
Lower Upper

$\begin{array}{lllll}0.328 & 0.428 & 2.674 & 2.707 & 0.007\end{array}$

$\begin{array}{lllll}0.278 & -0.420 & 1.648 & 1.163 & 0.245\end{array}$

$\begin{array}{llllll}0.115 & 0.428 & 1.757 & 3.224 & 0.001\end{array}$

$\begin{array}{llllll}0.065 & 0.571 & 1.572 & 4.197 & 0.000\end{array}$

$\begin{array}{lllll}0.201 & -0.638 & 1.121 & 0.538 & 0.590\end{array}$

$\begin{array}{llllll}0.237 & -2.167 & -0.260 & -2.494 & 0.013\end{array}$

$\begin{array}{lllll}0.109 & -1.074 & 0.219 & -1.295 & 0.195\end{array}$

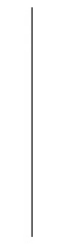

$-4.00$
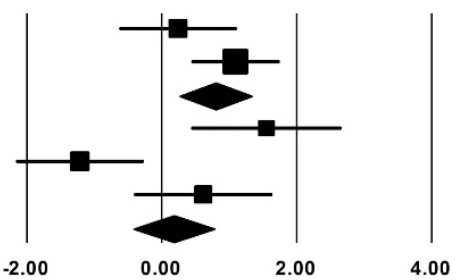
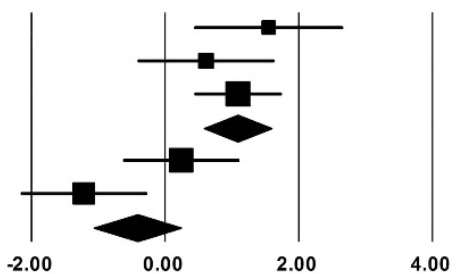

C

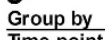

Study name

Time point

\begin{tabular}{|c|c|c|c|c|c|c|}
\hline \multicolumn{7}{|c|}{ Statistics for each study } \\
\hline $\begin{array}{l}\text { Std diff } \\
\text { in means }\end{array}$ & $\begin{array}{l}\text { Standard } \\
\text { error }\end{array}$ & Variance & $\begin{array}{r}\text { Lower } \\
\text { limit }\end{array}$ & $\begin{array}{c}\text { Upper } \\
\text { limit }\end{array}$ & Z-Value & p-Value \\
\hline 1.551 & 0.573 & 0.328 & 0.428 & 2.674 & 2.707 & 0.007 \\
\hline-1.214 & 0.487 & 0.237 & -2.167 & -0.260 & -2.494 & 0.013 \\
\hline 141.093 & 0.339 & 0.115 & 0.428 & 1.757 & 3.224 & 0.001 \\
\hline 0.570 & 0.250 & 0.063 & 0.080 & 1.061 & 2.279 & 0.023 \\
\hline 0.242 & 0.449 & 0.201 & -0.638 & 1.121 & 0.538 & 0.590 \\
\hline 0.614 & 0.528 & 0.278 & -0.420 & 1.648 & 1.163 & 0.245 \\
\hline 0.398 & 0.342 & 0.117 & -0.272 & 1.068 & 1.164 & 0.245 \\
\hline
\end{tabular}
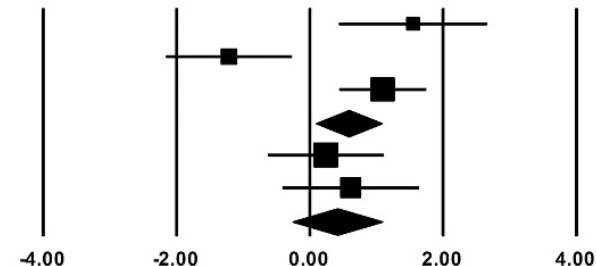

Figure 3 Subanalysis. (a). Forest plot examining the effects tDCS on reducing pain intensity based on inclusion or exclusion of participants with depressive symptoms. (b) Forest plot comparing the effects tDCS on reducing pain intensity based on average duration of pain among participants. (c) Forest plot examing the effects tDCS on reducing pain intensity based on duration of treatment. Note: Std diff: standard difference. 
in the reduction of NP. Furthermore, the study showed that the pooled effect of tDCS did not quite reach clinical importance. Minimal clinically important difference (MCID) in pain reduction on a numerical analog scale $(0-10)$ ranges from $\sim 1.5^{23}$ to $1.8,{ }^{24}$ whereas the current meta-analysis found a pooled decrease of 1.33 units at end of treatment. These findings are consistent with previous reviews examining the effect of tDCS among chronic pain ${ }^{25}$ and multiple sclerosis ${ }^{26}$ populations.

The sub-analysis examining duration of treatment found that shorter duration of treatment $(<1$ week) resulted in a significant decrease in pain intensity compared with a longer duration ( $>1$ week). Boggio et al. ${ }^{27}$ also found that weekly sessions of tDCS were no better than a single tDCS session among individuals with stroke. The mechanism of tDCS is complex and poorly understood. It involves weak electrical currents designed to increase the excitability of pain modulating areas in the brain in order to decrease cortical excitability. ${ }^{12}$ It may be hypothesized that the introduction of weak electrical currents stimulates pain modulation early in the treatment phase; however, delivery of weak currents over a longer period of time may reduce responsiveness among some individuals. However, further research needs to be conducted in order to understand its mechanism better. Luedtke et al. ${ }^{25}$ found healthy volunteers responded negatively to tDCS compared with those with chronic pain, suggesting that the effectiveness of tDCS may be dependent upon a specific population or medical condition. Further investigation may be warranted among individuals with SCI.

Studies have linked increased rates of comorbid depression and psychological distress with long-term chronic pain. ${ }^{28}$ In our study, indirect comparison of the effectiveness of tDCS based on duration of pain found that those with neuropathic pain duration of $<5$ years were more likely to demonstrate a reduction in pain intensity compared with those with pain duration of $>5$ years. Therefore, among those with SCI, there may be an effect of time since pain onset on pain intensity. Further, the pooled analysis also found that those studies that excluded individuals with high depressive symptoms were more likely to respond to tDCS treatment than those that included them. Among individuals with longer pain duration and high psychological distress, the use of more multimodal treatment options may be recommended.

As all studies allowed participants to continue their pharmacological treatment protocol during the study, the concomitant drug treatment may have influenced the effectiveness of tDCS. Liebetanz et al. ${ }^{29}$ found that carbamazepine can decrease the effects of anodal stimulation. However, the studies included for analysis did not report what types of pharmacological medications were being used by participants. Future studies should examine the effect of drug interaction in the reduction in pain during tDCS treatment.

There are some limitations of the current meta-analysis that may influence the generalizability of results. First, only a limited number of studies $(n=5)$ met inclusion criteria. Furthermore, calculations of numbers needed to treat and numbers needed to harm were not possible as only two studies reported enough data. ${ }^{12,21}$ Selected studies had a lack of follow-up; hence, duration of the effect of tDCS in reducing pain could not be assessed, along with its long-term effects. It is important to note that pain is multidimensional and influenced by several factors. Among the studies selected, there was a lack of availability of related outcomes such as pain disability, quality of life or other psychosocial factors. Turk et al. ${ }^{30}$ developed the Initiative on Methods, Measurement and Pain Assessment in Clinical Trials recommendations for the core domains related to chronic pain. The group recommended that trials examining chronic pain should assess for six domains including: pain, physical functioning, emotional functioning, participant ratings of improvement, symptoms and adverse events and participant adherence to treatment. To provide a more comprehensive overview on the effect of tDCS on the neuropathic pain among SCI individuals, future studies should aim to include outcomes from the six domains.

\section{CONCLUSION}

In conclusion, the current meta-analysis found a moderate effect size of tDCS in reducing neuropathic pain among individuals with SCI. However, it did not reach MCID recommendations for pain reduction. Many factors including: length of treatment, duration of pain, presence of depressive symptoms and concomitant drug therapy, may influence the effectiveness of tDCS in reducing pain. The study was limited by the small number of trials $(n=5)$. Furthermore, lack of adequate follow-up did not allow for determination of long-term effects. Future studies should examine and report the effect of tDCS on several domains of pain. Trials examining sub-analysis of factors that may influence tDCS effectiveness are recommended.

\section{CONFLICT OF INTEREST}

The authors declare no conflict of interest.

\section{ACKNOWLEDGEMENTS}

We acknowledge Ontario Neurotrauma Foundation and Rick Hansen Institute for their continued support.

1 Treede RD, Jensen TS, Campbell JN, Cruccu G, Dostrovsky JO, Griffin JW et al. Neuropathic pain: redefinition and a grading system for clinical and research purposes. Neurology 2008; 70: 1630-1635.

2 Finnerup NB. Pain in patients with spinal cord injury. Pain 2013; 154: S71-S76.

3 Siddall PJ, McClelland JM, Rutkowski SB, Cousins MJ. A longitudinal study of the prevalence and characteristics of pain in the first 5 years following spinal cord injury. Pain 2003; 103: 249-257

4 Bonica JJ. Introduction: semantic, epidemiologic, and educational issuers. In: Casey KL (ed.). Pain and Central Nervous System Disease: The Central Pain Syndromes. Raven Press:: NY, USA, 1991

5 Margot-Duclot A, Tournebise H, Ventura M, Fattal C. What are the risk factors of occurence and chronicity of neuropathic pain in spinal cord injury patients? Ann Phys Rehabil Med 2009; 52: 111-123.

6 Dworkin RH, O'Connor AB, Backonja M, Farrar JT, Finnerup NB, Jensen TS et al. Pharmacologic management of neuropathic pain: evidence-based recommendations. Pain 2007; 132: 237-251.

7 Gordon DB, Love G. Pharmacologic management of neuropathic pain. Pain Manag Nurs 2004; 5: 19-33.

8 Nicholson BD. Evaluation and treatment of central pain syndromes. Neurology 2004 62: $\mathrm{S} 30-\mathrm{S} 36$.

9 Siddall PJ, Loeser JD. Pain following spinal cord injury. Spinal Cord 2001; 39: 63-73.

10 Wallace BA, Ashkan K, Benabid AL. Deep brain stimulation for the treatment of chronic, intractable pain. Neurosurg Clin N Am 2004; 15: 343-357.

11 Knechtel L, Thienel R, Schall U. Transcranial direct current stimulation: Neurophysiology and clinical applications. Neuropsychiatry 2013; 3: 89-96.

12 Fregni F, Boggio PS, Lima MC, Ferreira MJ, Wagner T, Rigonatti SP et al. A shamcontrolled, phase II trial of transcranial direct current stimulation for the treatment of central pain in traumatic spinal cord injury. Pain 2006; 122: 197-209.

13 García-Larrea L, Peyron R, Mertens P, Lavenne F, Le Bars D, Convers P et al. Electrical stimulation of motor cortex for pain control: a combined PET-scan and electrophysiological study. Pain 1999; 83: 259-273.

14 Reidler JS, Mendonca ME, Santana MB, Wang X, Lenkinski R, Motta AF et al. Effects of motor cortex modulation and descending inhibitory systems on pain thresholds in healthy subjects. J Pain 2012; 13: 450-458.

15 Moseley AM, Herbert RD, Sherrington C, Maher CG. Evidence for physiotherapy practice: a survey of the Physiotherapy Evidence Database (PEDro). Aust J Physiother 2002; 48: 43-49.

16 Foley NC, Bhogal SK, Teasell RW, Bureau Y, Speechley MR. Estimates of quality and reliability with the physiotherapy evidence-based database scale to assess the methodology of randomized controlled trials of pharmacological and nonpharmacological interventions. Physical Therapy 2006; 86: 817-824.

17 Cohen J. Statistical Power And Analysis for Behavioral Sciences. Academic Press: New York, 1988 
18 Lipsey M, Wilson D. Practical Meta-Analysis. SAGE Publications Inc.: Thousand Oaks, CA, USA, 2001

19 Soler MD, Kumru H, Pelayo R, Vidal J, Tormos J, Fregni F et al. Effectiveness of transcranial direct current stimulation and visual illusion on neuropathic pain in spinal cord injury. Brain 2010; 133: 2565-2577.

20 Ngernyam N, Jensen MP, Arayawichanon P, Auvichayapat N, Tiamkao S, Janjarasjitt S et al. The effects of transcranial direct current stimulation in patients with neuropathic pain from spinal cord injury. Clin Neurophysiol 2014; 126: 382-390.

21 Wrigley PJ, Gustin SM, McIndoe LN, Chakiath RJ, Henderson LA, Siddall PJ. Longstanding neuropathic pain after spinal cord injury is refractory to transcranial direct current stimulation: A randomized controlled trial. Pain 2013; 154: 2178-2184.

22 Yoon EJ, Kim YK, Kim HR, Kim SE, Lee Y, Shin HI. Transcranial direct current stimulation to lessen neuropathic pain after spinal cord injury: a mechanistic PET study. Neurorehabil Neural Repair 2014; 28: 250-259.

23 Ostelo RW, Deyo RA, Stratford P, Waddell G, Croft P, Von Korff M et al. Interpreting change scores for pain and functional status in low back pain: towards international consensus regarding minimal important change. Spine 2008; 33: 90-94.

24 Hanley M, Jensen M, Ehde D, Robinson L, Cardenas D, Turner J et al. Clinically significant change in pain intensity ratings in persons with spinal cord injury or amputation. Clin J Pain 2006; 22: 25-31.
25 Luedtke K, Rushton A, Wright C, Geiss B, Juergens TP, May A. Transcranial direct current stimulation for the reduction of clinical and experimentally induced pain: $A$ systematic review and meta-analysis. Clin J Pain 2012; 28: 452-461.

26 Mori $\mathrm{F}$, Codecà $\mathrm{C}$, Kusayanagi $\mathrm{H}$, Monteleone $\mathrm{F}$, Buttari $\mathrm{F}$, Fiore $\mathrm{S}$ et al. Effects of anodal transcranial direct current stimulation on chronic neuropathic pain in patients with multiple sclerosis. J Pain 2010; 11: 436-442.

27 Boggio PS, Nunes A, Rigonatti SP, Nitsche MA, Pascual-Leone A, Fregni F. Repeated sessions of noninvasive brain DC stimulation is associated with motor function improvement in stroke patients. Restor Neurol Neurosci 2007; 25: 123-129.

28 Lynch ME, Campbell FA, Clark AJ, Dunbar MJ, Goldstein D, Peng P et al. Waiting for treatment for chronic pain-a survey of existing benchmarks: Toward establishing evidence-based benchmarks for medically acceptable waiting times. Pain Res Manag 2007; 12: 245.

29 Liebetanz D, Nitsche MA, Tergau F, Paulus W. Pharmacological approach to the mechanisms of transcranial DC-stimulation-induced after-effects of human motor cortex excitability. Brain 2002; 125: 2238-2247.

30 Turk DC, Dworkin RH, Allen RR, Bellamy N, Brandenburg N, Carr DB et al. Core outcome domains for chronic pain clinical trials: IMMPACT recommendations. Pain 2003; 106: 337-345. 